\title{
Eco-aesthetics: nurturing nature in the education of spatial design
}

\author{
R. Reed \\ The University of North Texas, USA
}

\begin{abstract}
Providing opportunities to investigate new biophilic forms in architecture and interior design rather than taking a human centred approach to design redirects beginning design students' imagination towards a nature-centred approach with the freedom to explore creative approaches to design using natural forms. In this methodology, the focus on design aesthetics provided by nature can be examined more closely void of traditional design constraints. Additionally, nature influences and fosters new methods for academicians with the utmost goal to build a greater awareness, sensibility, appreciation and placed value upon nature as a form-giver in the built environment. A series of spatial exercises were assigned using natural forms selected by the students. Biophilic and fractal design patterns were analyzed, sketched, and modelled in prototype form. Lastly, each student generated physical models of architectural structures, interior space plans and product design, which might include furniture, lighting, and other consumer oriented products, each resulting from patterns observed using the natural forms. This theoretical approach to teaching beginning space design provides a platform for students to draw from during advanced design studios. From these exercises, students were able to generate design solutions using natural forms more efficiently and quickly; most importantly, the results were far more creative as a result of looking outside for design inspiration inside.
\end{abstract}

Keywords: biophilic design, fractal patterns, nature, interior design, pedagogy.

\section{Introduction}

The natural environment has been a source of wonder and inspiration for millennia. It is not uncommon to see our natural environment as a catalyst for the production and use of everyday objects to connect on a physical, emotional, 
and intellectual level. Kellert [1] suggests that interaction with nature is necessary for our well-being and physical and mental development. The human condition to connect to our natural surroundings manifests itself in the visual consumption of our environment realized in the design of products and shelter. Plato, Vitruvius, and Aristotle were all founders of design based on organic, natural forms and structures. Egyptians and Ancient Greek civilizations studied nature along with the human body to abstract these forms into harmonious geometric proportions according to Pearson [2]. Flannery [3] states that "we have become more separated from the natural world, the value of what contact we do have, and minimal though it may be and often in the form of representations, becomes more important."

Objects are artefacts of the human condition; they reflect historical changes in technology, shifts in cultural and political views, socio-economic changes, trends and fads, all working together to transcend design to become part of our history. Post World War II, advancement in techniques for creating products expanded greatly with moulded forms, single injected moulded plastic shapes, bent tubular steel and plywood. Historically, references to natural forms in design and architecture have been decorative and cosmetic applications traditionally used as ornamental motifs whereas modern design continues to focus on experimenting with natural form, shape, and fractal patterns. This new technology allows designers to express themselves in ways they previously could not. With a sense of environmental urgency, finding more ecological and sustainable design process made nature not surprisingly, a clear source to explore new bio-inspired design solutions.

Nature's influence on product design and human behaviour is evident from its vast use within architecture, landscape architecture, and the decorative arts. Design shapes behaviour, behaviour shapes culture, and culture shapes design. Our world in ever changing, and design is ever evolving. "What is true of the chair is true of all the artefacts we create...we design them; but once built, they shape us" says Cranz [4]. Nature influences design and in turn design becomes a by-product realized from natural expression whereby nature proceeds to shape human behaviour through its design. When we select a certain object for use, we have inadvertently shaped our behaviour relative to the object's design. Take the chair for instance, probably the most widely used object on a day to day basis and probably the one item most taken for granted. The shape, slope, curve, softness and hardness all play a role in how the human body performs during sitting. Whether we recline, sit with our legs crossed, fidget from lack of comfort, or rock in constant motion, these behaviours are a direct result of the object's design.

\section{Design theory}

Design can be considered intention, purpose, communication, reaction, beauty, meaning, and influential in shaping our lives. The process of designing suggests in itself a set of guiding laws or principles for examining the success of new concepts and ideas. Beginning design students are introduced to a core of design 
values referred to as the elements and principles. These two categories hold the basic concepts for communication within all design professions and are the tools for the creation and design of all things.

Pentti Routio [5] identifies specific goals that all design should acquire; Usability - utility and function; Beauty - aesthetics (principles and elements of design); Meaning - messages sent; Ecology - impact to the environment; Economy - value and price; and Safety. Design is an experience through all of our senses meeting all of our basic human needs. Our relationship to art, architecture, and interior design is a personal one based on the way we perceive our surroundings and our natural environment. Beginning design students must understand how to use the elements of design in coordination with the principles of design for the outcomes to be effective, appropriate, and creative.

\section{Natural forms and design}

Nature in itself is a form of non-verbal communication that transcends all cultures. Holistically, it is the purist element to derive inspiration from and to be used in the development of product for human consumption. Natural symbols are culturally specific and in-turn possesses a greater meaning and social significance. For instance, consider the use of the bald eagle in American culture or the crane in Japanese culture. The use of these and other forms are not only culturally specific, but geographical as well. A pine cone from the forest or a nautilus shell from the ocean may lack regional appeal for someone residing in an arid, desert region. As both symbol and metaphor, objects inspired from nature draw deeper personal connection to relate to the everyday in a way that creates more meaning. Objects designed from the use of nature can develop meaning beyond its intended purpose creating narrative and story of its creation thereby forming more interest and personal connection to the individual user. This has the potential for nature applied design to become an outward expression of a specific person, place, or culture.

From the jagged lines of mountain top to the simple curves of a calalily; organic, natural design is all around us. Interior designers and architects seek inspiration from many sources. Natural forms have influenced the work of architects Frank Lloyd Wright, William Morris, Antonio Gaudi and artist Leonardo De Vinci. We draw inspiration and examples from nature into the design of a simple chair to a skyscraper using recognizable forms allowing the human experience to immediately make an identifiable connection to the object or space being experienced.

The basic components of all design are simple shapes. Rectangles, circles, squares, and triangles are basic shapes utilized in design. Frank Lloyd Wright drew inspiration from the hollyhock flower for his design of the 1921 Hollyhock House in Los Angeles, California. His refinement of the natural form into basic geometric shapes illustrates the flexibility of nature in creating new forms. The iconic Egg chair designed in 1958 by Arne Jacobsen is an example of the modernistic approach to using simple forms. Jacobsen carved out the desired shape for his chair creating interplay of positive and negative space while 
maintaining the simplicity of the original form. This same concept is evident in the Ear chair, 1968, by Georges Laporte and the Tongue chair Designed by Pierre Paulin in 1967. These examples suggest the use of human forms in addition to natural forms.

Nature selected for design provides a source of symbolic connection to place and context, reflection of cultural attitudes and beliefs, and exploration of design through new technologies and materials. The Bahá'i Temple in New Delhi is a pure geometric form representing a giant lotus flower [7]. The meaning of this symbol represents the spiritual connection the lotus flower has to the Baháí faith. Beyond nature as metaphor, using natural forms creates a sense of justification and relevance to an object that may not have existed otherwise and making its intended purpose more visible. If a design is overly simplified or even complicated nature becomes a source of recognition drawing connection with natural qualities which in turn provides meaning that explains and reasons the design to the user. Using nature in this manner is intended to explain a design which is otherwise confusion and often times not understood. "Nature... tends to organize growth patterns into this sequence...therefore; we automatically judge something in nature as aesthetically pleasing or beautiful" Reed [8].

As technology changes, ideas change, and so do the products we buy and the behaviours resulting from their use. Objects are symbols of social status and often used for expression of current social and cultural norms. When designers communicate through the use of materials in a new way, they may be expressing new ideas, concepts and establishing future perspectives for the advancement of their personal beliefs and often of their culture. Cranz [5] states that "The implicit theory is that the design line, proportions, shapes, and decorative motifs of the time crystallize the concerns and aspirations of the day."

\section{Design interprets nature}

Circular repetitive forms of a shell, the angular and rhythm of a palm frond, or the radial symmetry in the petals of a flower are examples of natural pattern forms. These patterns exist in every natural form whether human, animal, or plant. Richard Dubé [6] states that "when a designer chooses a pattern form on the basis of aesthetics, the choice will likely be driven by one or more of the following factors: emotions, scale, texture, or broad applicability."

So powerful is design and its impact on our day to day living that comprehending a holistic perspective of what design is, especially as a new design student, can be all but impossible. Students learn by rules, either established classroom requirements, or a set of steps to achieve success to a project. Students, as learners of new knowledge look for advice, for direction, for a source to guide them in their educational path to this knowledge. However, rules can be stifling, offering little to the imagination for a designer or artist. Building solid foundations that allow students creative freedom to explore and experiment with new design knowledge requires a source for guiding these initial investigations. Enter nature. 


\section{Nature in the design studio}

Beginning design students may find it difficult to grasp basic design theory as it applies to the creation of product and shelter. During a first year introductory course on design theory a project was developed using natural forms for design exploration. The purpose of the project was to use an active learning approach for instructional delivery to generate a greater comprehension of this new knowledge according to Bonwell and Eison [9]. This learning style when applied to a first year design course sought to strengthen the students learning from the traditional lecture method. The intent of the project was to provide process based learning using multiple techniques for design inquiry to form. Acquisition of the new knowledge included methods of observation and analysis, sketching, modelling, and writing to approach learning the principles of design defined by Kilmer and Kilmer [10] as harmony, unity \& variety, scale, proportion, emphasis, rhythm, balance and the elements of design; space, line, shape, form, texture, colour and light. Through these multiple modes of inquiry students would be able to form a deeper understanding and connection to design theory at an earlier stage in their education. Approaching beginning design in this manner, using the elements and principles, helps to find the right balance of both creativity and function while in turn introducing style and aesthetic importance to the final outcomes. If we break natural forms down into the basics for analysis, then application has the potential to be much easier and understandable for the student in applying theory to solve complex interior and architectural problems simultaneously generating an awareness and appreciation for bio-inspired and eco-friendly design.

\subsection{The process}

The deliverables of the design project involved the application of design principles and theory through exploration of natural forms into a three dimensional object for human use. The design process consisted of three phases: (1) object selection, (2) 2-D sketch investigation, (3) 3-D model prototyping and (4) final design solution implementation.

\subsubsection{Phase 1: natural object identification and selection}

The selection requires students to openly consider and closely examine design in nature where complex forms, shapes, textures, and growth patterns can provide an opportunity to investigate basic design principles. Examples of these forms might include a dandelion seed, a peacock feather, a spider's web, or the human body. Students are discouraged to consider forms that are limited in visual expression. If the object is too simple, such as a smooth rock, it may be difficult to execute.

\subsubsection{Phase 2: concept development and sketching}

In the second phase, students were asked to explore the object through analysis of the object's markings, patterns, shapes, textures, forms, and proportions from all observational angles and sides. These observations generated a series of 
study sketches, or visual biological language, which provides source material for the development of the newly created object or space later in the process. The students must generate plan, elevation, section, perspective and enlarged and sketch close-up views of the object as necessary. Additionally, sketches should capture texture, light, shadow, and shape.

Observed patterns were combined through continued sketching executing new patterns providing an opportunity for the students to explore additional design possibilities. Through this process, students were able to dissect the original form into its basic geometric and organic shapes refining the object while maintaining a sense of the original form. Figure 1 illustrates a series of sketches of the common dandelion seed shape and peacock bird feather exploring and narrating expressions of forms and patterns from the student's chosen natural object.

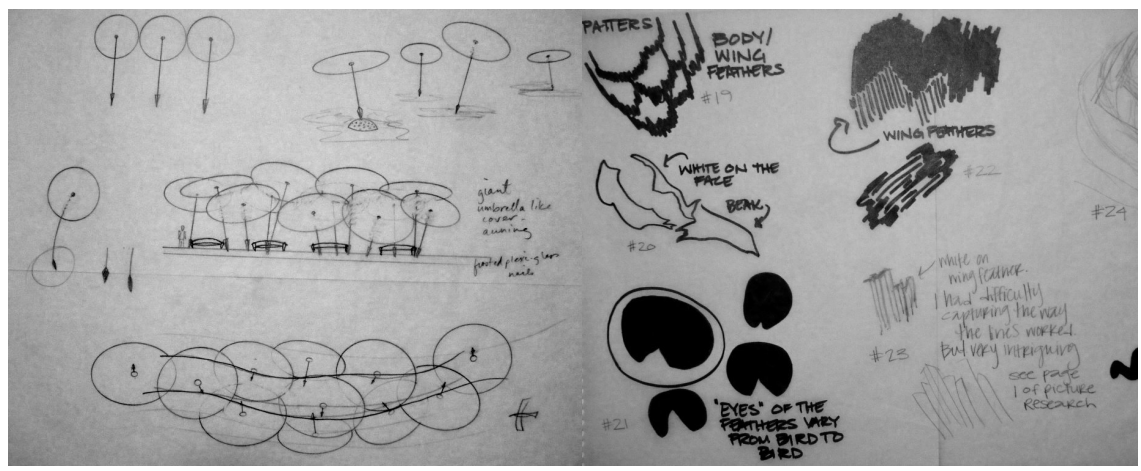

Figure 1: Initial patterns sketched to explore the various configurations of a dandelion seed shape and form (left) and the eye of a peacock bird feather (right).

\subsubsection{Phase 3: 3-D modelling and prototyping}

Students generate an extensive collection of visual notations and bio-inspired graphic language assembled into a conceptual document of the design process. Once a clear concept of the structure of object to be created emerges, a series of study models are developed to explore the $3^{\text {rd }}$ dimension while learning to translate and apply the principles of design visually. These models were encouraged to be an "abstracted" form, not literal of the original natural object, but rather simplified while maintaining the original natural form and combined, arranged into new forms as shown in Figure 2. The students may repeat each step of phase two (sketching and prototyping) until a desired solution is found.

In Figure 2 the students concentrated on the organic qualities and curvature of each of their chosen forms in three-dimensional model prototypes. Here they use any materials (paper, plastic, and cardboard, wire) to experiment with various solutions to their intended final structure or object to study nature as spatial form-givers. 


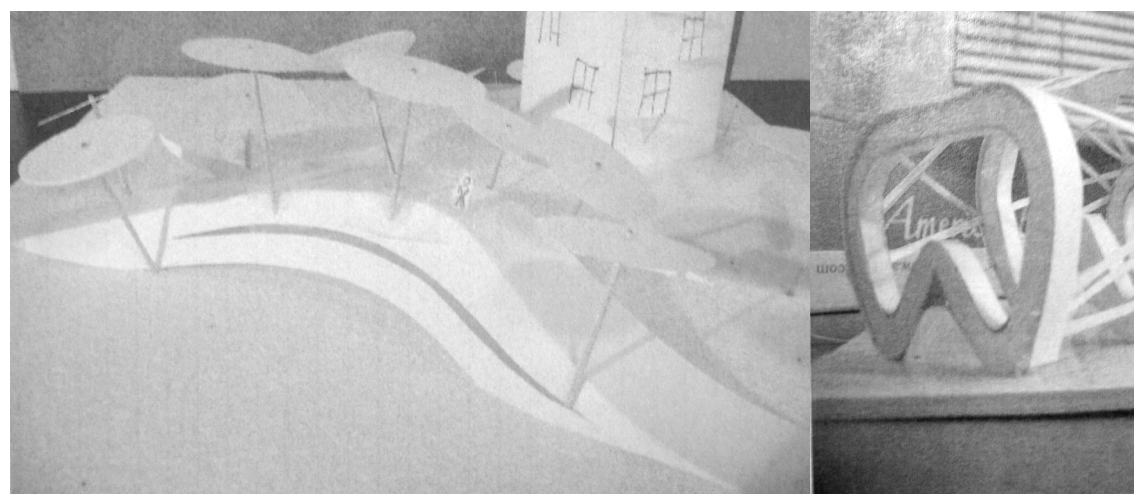

Figure 2: $\quad$ Prototyping initial concepts in three-dimensions as a result of visual pattern language generated in sketch studies.

\subsubsection{Phase 4: final design solution implementation}

After completion of the abstraction of the students' selected natural form they were asked to consider alternative purposes for the object. If this were to be manufactured for human use, what form could the object assume for practical use? Depending on the original object, student models resulted in the natural form lending itself to recognizable products such as a building, pedestrian walkway, lighting, furniture, etc.

During this phase additional study models may be generated to refine the natural form and explore additional design patterns. Once a final design has been identified and sketching near completion, a final production model representing the structure or object emerges, Figure 3.

In each solution, evidence can be seen for using nature to interpret basic design theory in beginning interior design courses. It should be noted in the initial project delivery, material selection was considered. Materials were chosen to represent the interplay of light and shadow, translucency and opacity further adding to the visual connection and recognizable cues of the design to the original organic form. The "aesthetic usability effect" so named by Lidwell et al. [11] suggests that the final design solution's aesthetic designs will be "perceived as easier to use than less-aesthetic designs." The results illustrate that students were able to maintain the integrity of the natural form-giver through creative use and interpretation of material and design.

\section{Conclusion}

In applying nature at the beginning stages of design education two factors emerge; (1) the aesthetic design opportunities nature provides for exploring basic design theory and (2) the elevated awareness and appreciation for nature earlier in the academic timeline whereby natures provides a basis for reasoning the design to the student and in doing so sets a precedence for natural forms leading 


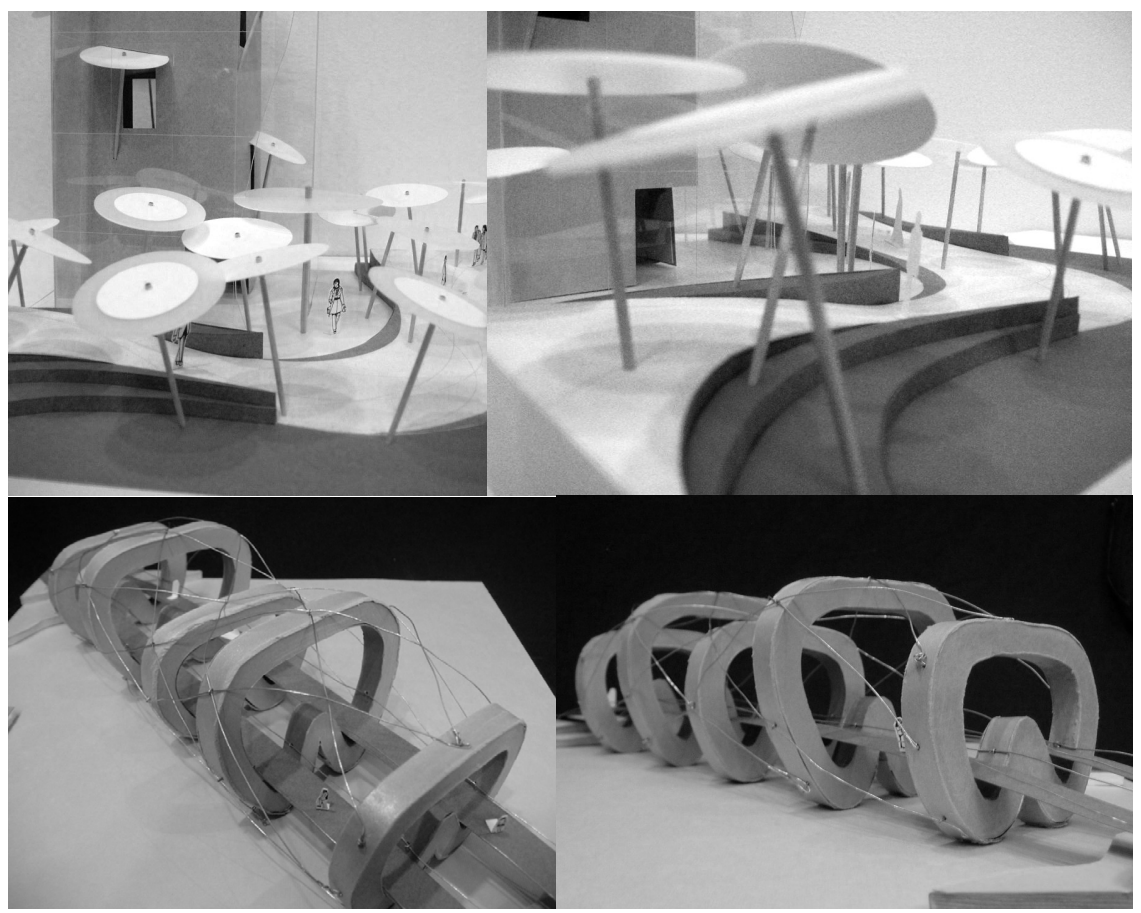

Figure 3: A series of final solutions including a pedestrian park with opaque and translucent areas of shade and cover based on the dandelion seed and a pedestrian bridge utilizing the eye of a peacock feather as the structural form.

to further advanced studies in eco and bio-inspired design. This theoretical approach to design positions itself in a less subjective manner strengthening the relevance of nature in the final solution.

Nature informs design. This work presents a point of consideration for possibilities of learning about design, through design, and the power to connect and generate ideas. Using nature as a source for inspiration and creativity allows for design realization to occur more fluidly. Students are exposed to alternative forms for exploring design and therefore retain a greater awareness through observation and exploration of uncommon forms tapping into the beauty of nature and its potential to influence design decisions.

In addition, the nature project creates a connection and sensitivity to nature that possibly leads students towards a deeper appreciation for green and sustainability design in later coursework. This process illustrates a method to incorporate active learning styles with source inspiration for teaching basic design theories to beginning interior design students. The nature project as pedagogical approach permitted better synthesis of knowledge and a broader base for understanding the principle of design resulting in a deeper understanding of design theory and approaches for design inquiry to occur. 


\subsection{Future implications}

Subsequent iterations of the natural form-givers design problem will include the additional design factor of physical and actual materiality vs. modelling tools to achieve experiential results. This addition will increase the students' awareness of social responsibility in the selection and application process of finishes and materials. Materiality presents an opportunity for students to consider natural evolutionary processes in formulating hypothetical outcomes as a factor in the potential performance evaluation of both the design with the material(s).

Finally, instilling respect and appreciation for what nature offers creates a deeper level of social awareness of natural forms among our youth. This in turn generates a greater sense of social responsibility significantly impacting the future of our built environment.

\section{References}

[1] Kellert, Stephen R., Building for Life: Designing and Understanding the Human-Nature Connection, Island Press: Washington, pp. 1-8, 2005.

[2] Pearson, David., New Organic Architecture: The Breaking Wave, University of California Press: Berkley, p. 30, 2001.

[3] Flannery, Maura C., Jellyfish on the Ceiling and Deer in the Den: The Biology of Interior Decoration. Leonardo, 38(3), pp. 239-244, 2005.

[4] Cranz, Galen., The Chair: Rethinking Culture, Body, and Design, W. W. Norton \& Company: New York, pp. 15 \& 69, 1998.

[5] Routio, Pentti., Theory of Design, http://membres.lycos.fr/routio/122.htm

[6] Dubé, Richard L., A Pattern Language: A Practical Source for Landscape Design, Van Nostrand Reinhold: New York, p. 63, 1997.

[7] Extracts from Interviews with the Architect, www.bahaindia.org/temple/ interview.html

[8] Reed, Ron., Color + Design: Transforming Interior Space, Fairchild: New York, p. 105, 2010.

[9] Bonwell, Charles C., \& James A. Eison., Active Learning: Creating Excitement in the Classroom, ASHE-ERIC Higher Education Report No. 1. The George Washington University, School of Education and Human Development: Washington, D.C., 1991.

[10] Kilmer, Otie W. \& Kilmer, Rosemary, Designing Interiors, Rinehart and Winston: Fort Worth, TX, pp. 88-121, 1992.

[11] Lidwell, William, Holden, Katrina, \& Butler, Jill, Universal Principles of Design, Rockport: Gloucester, MA, pp. 18-19, 2003. 